\title{
Surgical Oncology
}

National Cancer Institute

\section{Source}

National Cancer Institute. Surgical Oncology. NCI Thesaurus. Code C17839.

Intervention studies in which surgery is the dominant feature to prevent, diagnose, stage, or treat cancer (except neurosurgery and eye surgery). Includes special devices for dividing or destroying tissues, development and improvement of instruments for detection and diagnosis of tumors where surgery/biopsy is the dominant feature, cytoreductive procedures, intraoperative radiotherapy, surgical treatment of cancer and its complications, surgical supportive care, and plastic reconstructive and rehabilitation surgery. 